\title{
The Restrained Geodetic Number of a Line Graph
}

\author{
Ashalatha K. S. \\ Affiliated to Tumkur University \\ Department of Mathematics \\ Government First Grade College,Gubbi \\ Tumkur, Karnataka, India
}

\author{
Venkanagouda M. Goudar \\ Sri Siddhartha University \\ Department of Mathematics \\ Sri Siddhartha Institute of Technology \\ Tumkur, Karnataka, India
}

\begin{abstract}
For any graph $G(V, E)$, the line graph of $G$ denoted by $L(G)$. The Line graph $L(G)$ whose vertices corresponds to the edges of $G$ and two vertices in $L(G)$ are adjacent if and only if the corresponding edges in $G$ are adjacent. A geodetic set $S \subseteq V(G)$ of a graph $G=(V, E)$ is a restrained geodetic set if the subgraph $V-S$ has no isolated vertex. The minimum cardinality of a restrained geodetic set is the restrained geodetic number. In this paper we obtained the restrained geodetic number of line graph of any graph. Also, obtained many bounds on restrained geodetic number in terms of elements of $G$ and covering number of $G$.
\end{abstract}

\section{Keywords}

Cross product, Distance, Geodetic number, Line graph, Vertex covering number.

\section{INTRODUCTION}

In this paper we follow the notations of [6]. All the graphs considered here are finite, non trivial, undirected and connected. As usual $n=|V|$ and $m=|E|$ denote the number of vertices and edges of a graph $G$, respectively. For any graph $G(V, E)$, the Line graph $L(G)$ whose vertices correspond to the edges of $G$ and two vertices in $L(G)$ are adjacent if and only if the corresponding edges in $G$ are adjacent. The distance $d(u, v)$ between two vertices $\mathrm{u}$ and $\mathrm{v}$ in a connected graph $G$ is the length of a shortest $u-v$ path in $G$. It is well known that this distance is a metric on the vertex set $V(G)$. For a vertex v of $G$, the eccentricity $e(v)$ is the distance between $\mathrm{v}$ and a vertex farthest from $\mathrm{v}$. The minimum eccentricity among the vertices of $G$ is the radius, $\operatorname{rad} G$, and the maximum eccentricity is its diameter, $\operatorname{diam} G$. A $u-v$ path of length $d(u, v)$ is called a $u-v$ geodesic. We define $I(u, v)$ to be the set (interval) of all vertices lying on some $u-v$ geodesic of $G$, and for a nonempty subset $S$ of $V(G), I(S)=\bigcup_{u, v \in S} I(u, v)$.

A set $S$ of vertices of $G$ is called a geodetic set in $G$ if $I(S)=V(G)$, and a geodetic set of minimum cardinality is a minimum geodetic set. The cardinality of a minimum geodetic set in $G$ is called the geodetic number $g(G)$. A set of vertices $S$ in a graph $G$ is a restrained geodetic set if $S$ is a geodetic set and the subgraph $V-S$ has no isolated vertex. The minimum cardinality of a restrained geodetic set, denoted $g_{r}(G)$, is called the restrained geodetic number of $G$.
Now we define restrained geodetic number of line graph of a graph $G$. A set $S^{\prime}$ of vertices of $L(G)=H$ is called a restrained geodetic set in $H$ if $I\left(S^{\prime}\right)=V(H)$ and $V-S^{\prime}$ has no isolated vertex. A restrained geodetic set of minimum cardinality is the restrained geodetic number of $L(G)$ and is denoted by $g_{r}[L(G)]$. The cartesian product (or direct product) $X \times Y$ of two sets $X$ and $Y$ is the set of all possible ordered pairs whose first component is a member of $X$ and whose second component is a member of $Y$. A vertex $\mathrm{v}$ is an extreme vertex in a graph $G$, if the subgraph induced by its neighbors is complete. A vertex cover (edge cover) in a graph $G$ is a set of vertices (edges) that covers all edges (vertices) of $G$. The minimum number of vertices (edges) in a vertex cover (edge cover) of $G$ is the vertex cover number $\alpha_{0}(G)$ (edge cover number $\alpha_{1}(G)$ ).

For any undefined term in this paper, see [5],[6].

\section{MATHEMATICS SUBJECT CLASSIFICATION:05C05, $05 \mathrm{C} 12$}

\section{PRELIMINARY NOTES}

We need the following results to prove further results.

THEOREM 3.1 4. Every geodetic set of a graph contains its extreme vertices.

THEOREM 3.2 4. If $G$ is a non trivial connected graph of order $n$ and diameter $d$, then $g(G) \leq n-d+1$.

THEOREM 3.3 4. Let $G$ be a connected graph of order at least 3 , If $G$ contains a minimum geodetic set $S$ with a vertex of $G$ lies on some $x-w$ geodesic in $G$ for some $w \in S$, then $g(G)=$ $g\left(G \times K_{2}\right)$.

Proposition 3.4. The end edges of a tree $T$ are the extreme vertices of a line graph $L(T)$ of $T$.

PROPOSITION 3.5. For any tree $T$ with order $n$ and diameter $d, L(T)$ and $T$ have the same value of $n-d$.

\section{MAIN RESULTS}

THEOREM 4.1. For any tree $T$ that has at least three internal vertices and $k$ end-edges, $g_{r}[L(T)]=k$.

Proof. Let $S$ be the set of all extreme vertices of a line graph $L(T)$ of a tree $T$, by the theorem 2.1, $g_{r}[L(T)] \geq|S|$. On the other hand 
for an internal vertex $v$ of $T$, there exists $x, y$ of $T$ such that $v$ lies on the unique $x, y$ geodesic in $T$. The corresponding end edges of $T$ are the extreme vertices of $L(T)$. Thus $g[L(T)] \leq|S|$. Also every geodesic set $S^{\prime}$ of $L(T)$ must contain $S$ which is the unique minimum geodesic set, such that $V-S^{\prime}$ has no isolated vertices. Thus by the above argument $S^{\prime}$ is the minimal restrained geodetic set of $L(T)$. Hence $|S|=\left|S^{\prime}\right|=k$. There fore $g_{r}[L(T)]=k$.

COROLlary 4.2. For any path $P_{n}$ with $n$ vertices, $g_{r}\left[L\left(P_{n}\right)\right]=2$.

Proof. Clearly the set of two end-edges of a path $P_{n}$ is its unique restrained geodesic set. From Theorem 1, the result follows.

THEOREM 4.3. For any tree $T$ of order $n$ and diameter $d$, then $g_{r}[L(T)] \leq n-d+1$.

Proof. Let $T$ be a non trivial connected graph of order $n$ and diameter $d$, let $\mathrm{u}$ and $\mathrm{v}$ be vertices of $L(T)$, for which $d(u, v)=d$, let $u=v_{0}, v_{1}, \ldots, v_{d}=v$ be a $u-v$ path of length $d$. Now let $S=V[L(T)]-\left\{v_{1}, v_{2}, \ldots, v_{d-1}\right\}$, from the proposition 2.5, $I(S)=V[L(T)]$ and consequently $g_{r}[L(T)] \leq|S|=n-d+1$.

THEOREM 4.4. For cycle $C_{n}$ of order $n \geq 6$,

$$
g_{r}\left[L\left(C_{n}\right)\right]=\left\{\begin{array}{l}
2 \text { if } n \text { is even } \\
3 \text { if } n \text { is odd } .
\end{array}\right.
$$

Proof. The line graph $L\left(C_{n}\right)$ of a cycle $C_{n}$ is again a cycle. We have the following cases

Case i. Let $n$ be even.

The set of any two antipodal vertices is a restrained geodetic set of $L\left(C_{2 n}\right)$.

Case ii. Let $n$ be odd.

No two vertices form a restrained geodetic set, since there exists a 3 vertex restrained geodetic set. Thus

$$
g_{r}\left[L\left(C_{n}\right)\right]=\left\{\begin{array}{l}
2 \text { if } \mathrm{n} \text { is even } \\
3 \text { if } \mathrm{n} \text { is odd. }
\end{array}\right.
$$

THEOREM 4.5. If every non end-vertex of a tree $T$ is adjacent to at least one end vertex, then $g_{r}[L(T)] \leq\left\lceil n-\frac{k}{2}\right\rceil$, where $k$ is number of end vertices in $T$.

Proof. If $\operatorname{diam}(T) \leq 3$, then the result is obvious. Let $\operatorname{diam}(T)>$ 3 and $S^{\prime}=\left\{v_{1}, v_{2}, \ldots, v_{k}\right\}$ be the set of all end vertices in $T$ with $\left|S^{\prime}\right|=k$. Now without loss of generality, every end edge of $T$ are the extreme vertices of $L(T)$. Suppose $L(T)$ does not contain any end vertex then $S=\left\{u_{1}, u_{2}, \ldots, u_{i}\right\}$, where $S \subseteq V[L(T)]$, forms a geodetic sets of $L(T)$. Further if $L(T)$ contains at least one end vertex w, then the set $S \cup\{w\}$ forms a geodetic set $L(T)$. Therefore in all the cases, We obtain $|S \cup\{w\}| \leq\left\lceil n-\frac{\left|S^{\prime}\right|}{2}\right\rceil \Rightarrow$ $g[L(T)] \leq\left\lceil n-\frac{k}{2}\right\rceil$.

THEOREM 4.6. For any tree $T$, with $m$ edges, $g[L(T)] \leq$ $m-\frac{\alpha_{1}(T)}{2}+2$.

Proof. Suppose $S^{\prime}=\left\{e_{1}, e_{2}, \ldots, e_{m}\right\}$ be the set of all end edges in $T$. Then $S^{\prime} \cup J$ where $J \subseteq E(T)-S^{\prime}$, be the minimal set of edges which covers the vertices of $T$ which is not covered by $S^{\prime}$, such that $\left|S^{\prime} \cup J\right|=\alpha_{1}(T)$. Now without loss generality in $L(T)$, let $I=\left\{u_{1}, u_{2}, \ldots, u_{i}\right\} \subseteq V[L(T)]$ be the set of all vertices in $L(T)$ formed by the end edges in $T$ is the minimal geodetic set of $L(T)$. Clearly it follows that $g[L(T)] \leq|E(T)|-\left|\left\lceil\frac{s^{\prime} \cup J}{2}\right\rceil\right|+2$ $\Rightarrow g[L(T)] \leq m-\frac{\alpha_{1}(T)}{2}+2$.
THEOREM 4.7. Let $G^{\prime}$ be the graph obtained by adding an end-edge $(u, v)$ to a cycle $C_{n}=G$ with $u \in G$ and $v \notin G$ then $g_{r}\left[L\left(G^{\prime}\right)\right]=3$, if $n$ is even, except for $C_{4}$.

Proof. Let $\left\{e_{1}, e_{2}, \ldots, e_{n}, e_{1}\right\}$ be a cycle with $n$ vertices which is even and let $G^{\prime}$ be the graph obtained from $G=C_{n}$ by adding an end-edge $(u, v)$ such that $u \in G$ and $v \notin G$. By the definition of line graph, $L\left(G^{\prime}\right)$ has $K_{3}$ as a induced subgraph, also the edge $(u, v)=e_{k}$ becomes a vertex of $L\left(G^{\prime}\right)$. Let $S=\left\{e_{k}, e_{i}, e_{j}\right\}$ are the vertices of $L\left(G^{\prime}\right)$ where $e_{i}, e_{j}$ are the edges incident on the antipodal vertex of $u$ in $G^{\prime}$, such that $I(S)=V\left[L\left(G^{\prime}\right)\right]$ and $V-S$ has no isolated vertices. Thus by the above argument $S$ is the minimal restrained geodetic set of $L\left(G^{\prime}\right)$. Therefore $g_{r}\left[L\left(G^{\prime}\right)\right]=$ 3 .

THEOREM 4.8. Let $G^{\prime}$ be the graph obtained by adding end edge $(u, v)$ to a cycle $C_{n}=G$ with $u \in G$ and $v \notin G$, then $g\left[L\left(G^{\prime}\right)\right]=2$, if $n$ is odd, except for $C_{3}$.

Proof. Let $\left\{e_{1}, e_{2}, \ldots, e_{n}, e_{1}\right\}$ be a cycle with $n$ vertices which is odd and let $G^{\prime}$ be the graph obtained from $G=C_{n}$ by adding an end edge $(u, v)$ such that $u \in G$ and $v \notin G$. By the definition of line graph, $L\left(G^{\prime}\right)$ has $\left\langle K_{3}\right\rangle$ as an induced sub graph, also the edge $(u, v)=e_{k}$ becomes a vertex of $L\left(G^{\prime}\right)$. Let $S=\left\{e_{k}, e_{i}\right\}$ be the set of vertices of $L\left(G^{\prime}\right)$, where $e_{i}=(a, b) \in G$ and $d(u, a)=d(u, b)$ in the graph $L\left(G^{\prime}\right)$, such that $I(S)=V\left[L\left(G^{\prime}\right)\right]$ and any vertex $e_{l} \in V-S, \operatorname{deg}\left(e_{l}\right) \neq 0$. Thus by the above argument $S$ is the minimal restrained geodetic set of $L\left(G^{\prime}\right)$. There fore $g_{r}\left[L\left(G^{\prime}\right)\right]=$ 2 .

THEOREM 4.9. Let $G$ ' be the graph obtained by adding endedge $\left(u_{i}, v_{i}\right), i=1,2, \ldots, n$ to each vertex of $G=C_{n}$ such that $u_{i} \in G, v_{i} \notin G$. Then $g_{r}\left[L\left(G^{\prime}\right)\right]=n$.

Proof. Let $\left\{e_{1}, e_{2}, \ldots, e_{n}, e_{1}\right\}$ be a cycle with $n$ vertices and $G=$ $C_{n}$. Let $G^{\prime}$ be the graph obtained by adding end edge $\left(u_{i}, v_{i}\right), i=$ $1,2,3, \ldots, n$ to each vertex of $G$ such that $u_{i} \in G, v_{i} \notin G$. Clearly $n$ be the number of end vertices of $G^{\prime}$. By the definition of line graph, $L\left(G^{\prime}\right)$ have $n$ copies of $K_{3}$ as an induced subgraph. The edges $\left(u_{i}, v_{i}\right)=e_{i}$ for all $i$, becomes $n$ vertices of $L\left(G^{\prime}\right)$ and those lies on geodetic set of $L\left(G^{\prime}\right)$. Since they forms the extreme vertices of $L\left(G^{\prime}\right)$, by Theorem 2.1. Which itself is the restrained geodetic set of $L\left(G^{\prime}\right.$. There fore $g_{r}\left[L\left(G^{\prime}\right)\right]=n$. $\frac{n}{\alpha_{0}\left(C_{n}\right)}$.

THEOREM 4.10. For cycle $C_{n}, n \geq 6$ is even, $g_{r}\left[L\left(C_{n}\right)\right]=$

Proof. Let $n \geq 6$ is even be the number of vertices and $\alpha_{0}$ is the vertex covering number of $G=C_{n}$. We have $L\left(C_{n}\right)=C_{n}$ and by Theorem 3.3, $g_{r}\left[L\left(C_{n}\right)\right]=2$. Also for even cycle, vertex covering number $\alpha_{0}\left(C_{n}\right)=\frac{n}{2}$. Hence $g_{r}\left[L\left(C_{n}\right)\right]=2=\frac{n}{\frac{n}{2}}=\frac{n}{\alpha_{0}\left(C_{n}\right)}$.

THEOREM 4.11. For cycle $C_{n}, n>5$ is odd, $g_{r}\left[L\left(C_{n}\right)\right]=$ $\frac{n+1}{\alpha_{0}\left(C_{n}\right)}+1$.

Proof. Let $n \geq 3$ is odd be the number of vertices and $\alpha_{0}$ is the vertex covering number of $G=C_{n}$. We have $L\left(C_{n}\right)=C_{n}$ and by Theorem 3.3, $g_{r}\left[L\left(C_{n}\right)\right]=3$. Also for odd cycle, vertex covering number $\alpha_{0}\left(C_{n}\right)=\frac{n+1}{2}$. Hence $g_{r}\left[L\left(C_{n}\right)\right]=2+1=\frac{n+1}{\frac{n+1}{2}}+1=$ $\frac{n+1}{\alpha_{0}\left(C_{n}\right)}+1$.

THEOREM 4.12. For any integers $m, n \geq 2$, $g_{r}\left[L\left(K_{m, n}\right)\right] \leq m n-1$. 
Proof. Let $m+n$ and $m n$ be the number of vertices and edges of the given graph $K_{m, n}$ and $d$ be the diameter. Since diameter of $L\left(K_{m, n}\right)=2$, the number of vertices in $L\left(K_{m, n}\right)$ is $m n$. Hence by Theorem $2.2 g_{r}(G) \leq n-d+1$. Now we have $g_{r}\left[L\left(K_{m, n}\right)\right] \leq$ $m n-2+1 \Rightarrow g_{r}\left[L\left(K_{m, n}\right)\right] \leq m n-1$.

THEOREM 4.13. For any integer $n \geq 4, g_{r}\left[L\left(K_{n}\right)\right] \leq$ $\frac{(n+1)(n-2)}{2}$.

Proof. Let $n \geq 4$ be the vertices of the given graph $K_{n}$ and $d$ be the diameter. Since diameter of $L\left(K_{n}\right)$ is 2 and the number of vertices in $L\left(K_{n}\right)$ is $\frac{n(n-1)}{2}$, hence by Theorem $2.2, g_{r}(G) \leq n-d+1$. We have $g_{r}\left[L\left(K_{n}\right)\right] \leq \frac{n(n-1)}{2}-2+1$.

$\Rightarrow g_{r}\left[L\left(K_{n}\right)\right] \leq \frac{n(n-1)}{2}-1$.

$\Rightarrow g_{r}\left[L\left(K_{n}\right)\right] \leq \frac{n^{2}-n-2}{2}$.

$\Rightarrow g_{r}\left[L\left(K_{n}\right)\right] \leq \frac{(n+1)(n-2)}{2}$.

THEOREM 4.14. For any path $P_{n}$,

$$
g\left[L\left(P_{n} \times K_{2}\right)\right]=\left\{\begin{array}{l}
2 \text { when } n=2 \\
3 \text { when } n=3 \\
4 \text { when } n>3
\end{array}\right.
$$

Proof. Let $P_{n} \times K_{2}$ be formed from two copies of $G_{1}$ and $G_{2}$ of $P_{n}$. Then by Theorem $2.3 g\left(P_{n} \times K_{2}\right)=g\left(P_{n}\right)$. Now $L\left(P_{n} \times K_{2}\right)$ formed from two copies of $G_{1}^{\prime}$ and $G_{2}^{\prime}$ of $L\left(P_{n}\right)$. And let $U=\left\{u_{1}, u_{2}, \ldots, u_{n-1}\right\} \in V\left(G_{1}^{\prime}\right)$, $W=\left\{w_{1}, w_{2}, \ldots, w_{n-1}\right\} \in V\left(G_{2}^{\prime}\right)$. We have the following cases.

Case 1: If $n=2$.

Then by the definition of line graph $L\left(P_{2} \times K_{2}\right)=P_{2} \times K_{2}$. By Theorem $2.3 g\left[L\left(P_{2} \times K_{2}\right)\right]=g\left(P_{2}\right)=2$.

Case2: If $n=3$.

Then $L\left(P_{3} \times K_{2}\right)$ is formed from two copies of $P_{2}$ clearly $g\left[L\left(P_{3} \times K_{2}\right)\right]=3$.

Case3: If $n>3$.

Let $S$ be the geodetic set of $L\left(P_{n} \times K_{2}\right)$. We claim that $S$ contains two elements (end vertices) from each set $\left\{u_{1}, u_{n-1}, w_{1}, w_{n-1}\right\}$. Since $I(S)=V\left[L\left(P_{n} \times K_{2}\right)\right]=V$, it follows that $g\left[L\left(P_{n} \times\right.\right.$ $\left.\left.K_{2}\right)\right] \leq 4$. It remains to show that if $S^{\prime}$ is a three element subset of $V\left[L\left(P_{n} \times K_{2}\right)\right]$ then $I\left(S^{\prime}\right) \neq V\left[L\left(P_{n} \times K_{2}\right)\right]$. First assume that $S^{\prime}$ is a subset $U$ or $W$, say the farmer. Then $I\left(S^{\prime}\right)=S^{\prime} \cup W \neq V$. Therefore, we may take that $S^{\prime} \cap U=\left\{u_{i}, u_{j}\right\}$ and $S^{\prime} \cap W=$ $\left\{w_{k}\right\}$. Then $I\left(S^{\prime}\right)=\left\{u_{i}, u_{j}\right\} \cup W \neq V\left[L\left(P_{n} \times K_{2}\right)\right]$.

THEOREM 4.15. For the wheel $W_{n}=K_{1}+C_{n-1}(n \geq 6)$, $n$ is even. Then $g_{r}\left[L\left(W_{n}\right)\right]=\frac{n}{2}$.

Proof. Let $W_{n}=K_{1}+C_{n-1}(n \geq 6)$ with $x$ the vertex of $K_{1}$ and $V\left(C_{n-1}\right)=\left\{v_{1}, v_{2}, \ldots, v_{n-1}\right\}, E=\left\{e_{1}, e_{2}, \ldots, e_{n-1}\right\}$ be the internal edges of $W_{n}$. Now, $U=\left\{u_{1}, u_{2}, \ldots, u_{j}\right\}$ are the vertices formed from edges of $C_{n-1}$ i.e $U \subseteq V\left[L\left(W_{n}\right)\right]$. $W=$ $\left\{w_{1}, w_{2}, \ldots, w_{j}\right\}$ are the vertices of $L\left(W_{n}\right)$ formed from internal edges of $W_{n}$, i.e $W \subseteq V\left[L\left(W_{n}\right)\right]$. Now $U_{1} \cup\left\{w_{j}\right\}$ where $U_{1} \subseteq U$, forms a minimum geodetic set of $L\left(W_{n}\right)$. Clearly $\left|U \cup\left\{w_{j}\right\}\right| \frac{n}{2}$. $\Rightarrow$ $g_{r}\left[L\left(W_{n}\right)\right]=\frac{n}{2}$.

THEOREM 4.16. For the wheel $W_{n}=K_{1}+C_{n-1}(n \geq 6)$, $n$ is odd. Then $g_{r}\left[L\left(W_{n}\right)\right]=\frac{n+1}{2}$.
Proof. Let $W_{n}=K_{1}+C_{n-1}(n \geq 6)$ with $x$ the vertex of $K_{1}$ and $V\left(C_{n-1}\right)=\left\{v_{1}, v_{2}, \ldots, v_{n-1}\right\}, E=\left\{e_{1}, e_{2}, \ldots, e_{n-1}\right\}$ be the internal edges of $W_{n}$. Now, $U=\left\{u_{1}, u_{2}, \ldots, u_{j}\right\}$ are the vertices formed from edges of $C_{n-1}$ i.e $U \subseteq V\left[L\left(W_{n}\right)\right]$. $W=\left\{w_{1}, w_{2}, \ldots, w_{j}\right\}$ are the vertices of $L\left(W_{n}\right)$ formed from internal edges of $W_{n}$, i.e $W \subseteq V\left[L\left(W_{n}\right)\right]$. Now $U_{1} \cup\left\{w_{j}, w_{j-1}\right\}$ where $U_{1} \subseteq U$, forms a minimum geodetic set of $L\left(W_{n}\right)$. Clearly $\left|U \cup\left\{w_{j}, w_{j-1}\right\}\right| \frac{n+1}{2} . \Rightarrow g_{r}\left[L\left(W_{n}\right)\right]=\frac{n+1}{2}$.

\section{REFERENCES}

[1] F Buckley and F. Harary. Distance in graphs, Addison-Wesely, Reading, MA (1990).

[2] G. Chartrand, F. Harary, and P.Zhang. Geodetic sets in graphs Discussiones Mathematicae Graph Theory 20 (2000), 129-138.

[3] G. Chartrand, F. Harary, H.C Swart and P.Zhang. Geodomination in graphs, Bull. ICA 31 (2001), 51-59.

[4] G. Chartrand, F. Harary, and P.Zhang. On the geodetic number of a graph.Networks.39 (2002) 1-6.

[5] G. Chartrand and P.Zhang. Introduction to Graph Theory, Tata McGraw Hill Pub.Co.Ltd.(2006).

[6] F.Harary, Graph Theory, Addison-Wesely, Reading, MA, 1969. 